\title{
Study of the risk factors related to acquisition of urinary tract infections in patients submitted to renal transplant
}

\author{
Mayra Gonçalves Menegueti ${ }^{[1]}$, Marcos Fernando Pereira ${ }^{[2]}$, \\ Fernando Bellissimo-Rodrigues ${ }^{[3]}$, Tania Marisa Pisi Garcia ${ }^{[2]}$, \\ Luciana Tanajura Santamaria Saber ${ }^{[2]}$, Maria Estela Papini Nardim ${ }^{[2],}$ \\ Valmir Aparecido Muglia ${ }^{[2]}$, Miguel Moyses Neto ${ }^{[2]}$ \\ and Elen Almeida Romão ${ }^{[2]}$
}

[1]. Comissão de Controle de Infecção Hospitalar, Hospital das Clínicas da Faculdade de Medicina de Ribeirão Preto, Universidade de São Paulo, Ribeirão Preto, São Paulo, Brasil. [2]. Divisão de Nefrologia, Faculdade de Medicina de Ribeirão Preto, Universidade de São Paulo, Ribeirão Preto, São Paulo, Brasil. [3]. Departamento de Medicina Social, Faculdade de Medicina de Ribeirão Preto, Universidade de São Paulo, Ribeirão Preto, São Paulo, Brasil.

\begin{abstract}
Introduction: Urinary tract infections (UTI) among transplant recipients are usually caused by gram-negative microorganisms and can provoke a high incidence of morbidity and mortality. The aim of this study was to evaluate the risk factors associated with the acquisition of UTIs during the first year after renal transplantation. Methods: Here, we report a single-center retrospective cohort study of 99 renal transplant patients followed for the first year after surgery. The definition of a UTI episode was a urine culture showing bacterial growth and leucocyturia when patients presented with urinary symptoms. The absence of infection (asymptomatic bacteriuria) was defined as an absence of symptoms with negative urine culture or bacterial growth with any number of colonies. Results: Ninety-nine patients were included in the study. During the study, 1,847 urine cultures were collected, and $320(17.3 \%)$ tested positive for bacterial growth. Twenty-six (26.2\%) patients developed a UTI. The most frequent microorganisms isolated from patients with UTIs were Klebsiella pneumoniae (36\%), with $33 \%$ of the strains resistant to carbapenems, followed by Escherichia coli (20\%). There were no deaths or graft losses associated with UTI episodes. Conclusions: Among the UTI risk factors studied, the only one that was associated with a higher incidence of infection was female sex. Moreover, the identification of drug-resistant strains is worrisome, as these infections have become widespread globally and represent a challenge in the control and management of infections, especially in solid organ transplantation.
\end{abstract}

Keywords: Urinary tract infections. Renal transplantation. Risk factors.

\section{INTRODUCTION}

Renal transplantation is an effective treatment for patients with advanced chronic renal disease, and the kidney is the most frequently transplanted organ. Although high mortality rates are observed in the first year post-transplantation, they are lower than those in patients undergoing dialysis with similar risk factor profiles; thus, transplantation can provide a better quality of life and increase the life expectancy ${ }^{(1)}$.

Urinary tract infections (UTI) are the most common infection after renal transplantation and are a major cause of morbidity

Corresponding author: Dra. Mayra Gonçalves Menegueti. CCIH/HC/FMRP/ USP. Av. Bandeirantes 3900, Bairro Monte Alegre, 14049-900 Ribeirão Preto, São Paulo, Brasil.

Phone/Fax: 55 16 3360-2319

e-mail: mayra_menegueti@yahoo.com.br

Received 19 March 2015

Accepted 20 May 2015 and mortality among transplant recipients. In a prospective study of 133 patients transplanted at a single center and followed for one year, 88 infections occurred in 60 patients, and UTIs were responsible for 46 of these cases $(53 \%)^{(2)}$. Another prospective study of 159 patients followed for two years after transplant showed a rate of 1.1 episodes of infection per patient, and UTIs were responsible for $46.6 \%$ of these episodes ${ }^{(3)}$.

Urinary tract infections among transplant recipients are usually caused by gram-negative microorganisms, of which Escherichia coli is the most prevalent. In a retrospective cohort study of 136 kidney transplant recipients, 56 developed UTIs, and the most commonly isolated microorganisms were $E$. coli $(59.1 \%)$ and Klebsiella spp. $(16.9 \%)^{(4)}$. In another retrospective cohort study of 393 kidney transplant recipients, 221 UTI episodes were reported, and the most commonly reported microorganisms were E. coli $(18.4 \%)$ and Klebsiella spp. $(14.6 \%)^{(5)}$.

The main risk factors for contracting a UTI related to kidney transplantation are urinary bladder catheterization, deceased donor kidney, abnormalities of the native kidney or the allograft (such as vesicoureteral reflux), use of a ureteral stent, 
diabetic neurogenic bladder, rejection episodes, and use of immunosuppressive drugs ${ }^{(6)(7)(8)(9)(10)}$.

The infections that occur in the hospital tend to be more severe, provoking bacteremia in $10 \%$ and allograft infection in $90 \%$ of recipients. These infections may be associated with allograft dysfunction and can predispose patients to the development of acute allograft rejection ${ }^{(11)(12)(13)}$.

The aim of this study was to evaluate the risk factors related to acquisition of UTIs in patients during the first year after renal transplantation.

\section{METHODS}

This study was conducted at the University Hospital of the Ribeirão Preto Medical School, University of São Paulo, Ribeirão Preto, SP, Brazil. It was a single-center retrospective cohort study of 99 renal transplanted patients (98 kidneys were obtained from deceased donors) followed during the first year after surgery. Demographic and transplant information and risk factors for UTI were collected from the medical records and the intranet system of the hospital. Renal function was evaluated by measuring plasma creatinine levels one year after kidney transplantation.

A UTI episode was defined as the presence of at least one of the following signs or symptoms: fever $\left(>38^{\circ} \mathrm{C}\right)$; suprapubic tenderness; costovertebral angle pain or tenderness; or a positive urine culture of $\geq 10^{5}$ colony-forming units (CFU)/mL with no more than 2 species of microorganisms ${ }^{(14)}$. UTIs were also diagnosed in patients $\leq 65$ years of age with fever $\left(>38^{\circ} \mathrm{C}\right)$, urgency, frequency, dysuria, suprapubic tenderness, costovertebral angle pain or tenderness and at least 1 of the following results: a) positive dipstick for leukocyte esterase and/or nitrite; b) pyuria (urine specimen with $\geq 10$ white blood cells $(\mathrm{WBC}) / \mathrm{mm}^{3}$ of unspun urine or $>5 \mathrm{WBC} /$ high-power field of spun urine; or c) microorganisms observed on Gram's stain of unspun urine and a positive urine culture of $\geq 10^{3}$ and $<10^{5}$ $\mathrm{CFU} / \mathrm{ml}$ with no more than 2 species of microorganisms ${ }^{(14)}$. The absence of infection was defined as negative urine cultures or bacterial growth with any number of colonies in the absence of symptoms.

Fresh voiding urine was preferably obtained for culture when feasible. For patients using urinary catheters, urine was aseptically obtained by puncturing the drainage system. The bacterial strains were identified using a semi-automated system (Vitek 2TM), which was also used for evaluating susceptibility to antimicrobial agents, according to the criteria of the Clinical Laboratory Standards Institute (CLSI) ${ }^{(15)}$.

Laboratory tests and urine cultures were programmed for each patient in a 2-week routine collection during hospital admittance. After discharge, the outpatient evaluation schedule was as follows: weekly for the first month, biweekly for the second month, and monthly beginning 3 months post-surgery. In the outpatient clinic, the urine cultures were performed every time the patient presented with symptoms suggestive of UTI and/or fever of unknown origin, allograft pain or leucocyturia even without symptoms. Loss of allograft was defined if the patient was submitted to nephrectomy or presented with allograft dysfunction requiring dialysis.

To analyze possible associations between clinical and demographic characteristics with the occurrence of UTI, we used a two-tailed Fisher's exact test. Means of continuous variables were compared using Wilcoxon's test for unpaired samples. To adjust the analysis for possible confounding factors, we used a logistic regression model that only included variables that presented an association with the occurrence of UTI. This was done by univariate statistical analysis with $p \leq 0.10$. The level of significance was set at $\alpha=0.05$ for all analyses.

\section{RESULTS}

Ninety-nine patients were included in the study. During the first year after renal transplantation, 20 patients lost their grafts, 10 patients were lost to follow up, and 7 patients died. None of the patients died or lost their graft as the direct result of a UTI during the study. Twenty-six (26.2\%) patients developed UTIs, ten of which were male and sixteen female. The mean time from the day of transplant surgery to the development of infection was 24 days (ranging from 5 to 102 days). The patients' median age was 50 years (ranging from 19 to 71 years). The median dialytic treatment period prior to transplantation was 48 months (ranging from 2 to 240 months), and $16 \%$ of patients showed vesicoureteral reflux or incomplete bladder emptying. Thirty-one percent of the patients were diabetic prior to transplantation. The cold ischemia median time for the deceased donors was 25 hours (ranging from 6 to 48 hours), and forty-two percent of the patients received thymoglobulin as an induction treatment. The only risk factor identified for UTI in the present study was female sex. The analysis of variables associated with the occurrence of UTI is shown in Table 1.

During the study, 1,847 urocultures were collected, and $320(17.3 \%)$ tested positive for bacterial growth. Seventeen cultures showed the presence of two microorganisms. Among the positive urine samples, $182(54.1 \%)$ presented less than $100,000 \mathrm{CFU} / \mathrm{mL}$, and $155(45.9 \%)$ presented more than $100,000 \mathrm{CFU} / \mathrm{mL}$. The identified microorganisms are shown in Table 2. The most frequently isolated microorganism in all urine cultures was Klebsiella pneumoniae (44.5\%), followed by E. coli $(15.4 \%)$. The $K$. pneumoniae strains were highly resistant to carbapenem antibiotics $(20.7 \%)$.

Of the 99 patients included in the study, 26 had a diagnosis of UTI according to the previous definition. The most frequently identified microorganisms responsible for UTIs were K. pneumoniae (36\%), E. coli (20\%), Pseudomonas aeruginosa (16\%) and Enterobacter cloacae (8\%). The antibiotic susceptibility profiles of the microorganisms are presented in Figure 1. The K. pneumoniae strains were highly resistant to carbapenem antibiotics (33\%). Two urine samples with E. cloacae, one with Enterococcus faecalis, and one with Acinetobacter baumannii showed susceptibility to all tested antibiotics. Burkholderia cepacia was susceptible to 
TABLE 1 - Clinical and demographic data from renal transplant recipients and the association with the occurrence of UTIs in the first year after kidney transplant. Ribeirão Preto, Brazil, 2014.

\begin{tabular}{|c|c|c|c|c|c|}
\hline & Total $(\mathrm{n}=99)$ & $\begin{array}{c}\text { UTI } \\
(n=26)\end{array}$ & $\begin{array}{l}\text { Without UTI } \\
\qquad(\mathrm{n}=73)\end{array}$ & $\begin{array}{c}\mathrm{p}^{*} \\
\text { univariated }\end{array}$ & $\begin{array}{l}\text { Regression } \\
\text { p-value }\end{array}$ \\
\hline Patients (\%) & 100.0 & 26.0 & 73.0 & - & - \\
\hline Female n $(\%)$ & $42(42.0)$ & $16(38.0)$ & $26(62.0)$ & 0.03 & 0.03 \\
\hline Age (years old) & 50 & 56 & 48 & 0.44 & \\
\hline Median (range) & $(2-240)$ & $(13-199)$ & $(2-240)$ & & \\
\hline Glomerulopathy prior to renal transplant n (\%) & $15(15.0)$ & $1(1.0)$ & $14(14.0)$ & 0.10 & 0.10 \\
\hline Vesicoureteral reflux n (\%) & $5(5.0)$ & $1(20.0)$ & $4(80.0)$ & 1.00 & \\
\hline Induction with thymoglobulin n (\%) & $42(42.0)$ & $11(26.0)$ & $31(74.0)$ & 1.00 & \\
\hline Diabetes mellitus n (\%) & $31(31.0)$ & $9(29.0)$ & $22(71.0)$ & 0.80 & \\
\hline Cold ischemia time (h) & 25 & 25 & 25 & 0.20 & \\
\hline Median (range) & $(1-41)$ & $(12-41 \mathrm{~h})$ & $(1-41)$ & & \\
\hline Infection of surgical site $\mathrm{n}(\%)$ & $7(7.0)$ & $1(14.0)$ & $6(86.0)$ & 0.18 & \\
\hline Antibiotic prophylaxis: 2 to 6 months n (\%) & $43(43.0)$ & $14(33.0)$ & $29(67.0)$ & & \\
\hline
\end{tabular}

UTI: Urinary tract infections. Analysis of the association among variables and UTI occurrence was performed using the two-tailed Fisher's exact test, and means of continuous variables were compared using Wilcoxon's test for unpaired samples. Multivariate analysis was performed by logistic regression.

ceftazidime, meropenem and sulfamethoxazole/trimethoprim. Candida albicans was susceptible to fluconazole. The most frequently used antibiotics for UTI treatment in the renal transplant patients were ciprofloxacin $(30 \%)$, meropenem $(26 \%)$, cefepime $(13 \%)$ and amikacin $(9 \%)$. The two patients with fungal UTIs were treated with fluconazole, and the patient who presented with a positive uroculture to E. faecalis was treated with ciprofloxacin.

\section{DISCUSSION}

The incidence of UTIs in the present study was $26.2 \%$, and the only risk factor identified was the female sex. In one prospective cohort multicenter study that enrolled 2,052 renal transplant patients, the incidence of UTIs was $10.04 \%$, and the independent risk factors were age, female sex and the need for dialysis post-transplantation ${ }^{(16)}$. Another report with 393 renal transplanted patients showed a UTI incidence of $56.23 \%$, and the only associated risk factor was age ${ }^{(5)}$. One retrospective study with 152 renal transplanted patients showed a UTI incidence of $46.71 \%$, and the associated risk factors were female sex, age less than 45 years old, and treatment with cyclosporine plus azathioprine and prednisolone ${ }^{(17)}$.
The most frequently isolated microorganisms in the present study were $K$. pneumoniae and E. coli. In contrast, other studies have shown a predominance of $E$. coli $(29 \%)$, followed by Enterococcus spp. $(24 \%)^{(18)}$. Souza et al. 2010 reported a predominance of E. coli (37\%), Enterobacter spp. (19\%) and Klebsiella spp. $(11 \%)^{(19)}$, and a multicenter study that included 1,762 urine samples collected from thirty-eight sites in eleven countries showed that the most frequent isolates were $E$. coli $(56.5 \%)$ and K. pneumoniae $(13.8 \%)^{(20)}$. In another prospective study, the authors followed 295 renal transplant patients up to four weeks post-transplantation and collected 582 urine cultures. Among the isolated bacterial strains $(n=291)$, the most common were gram-negative bacteria $(56.4 \%)$, predominantly Serratia marcescens $(32.3 \%)$, E. cloacae (14.6\%) and E. coli $(12.2 \%)^{(21)}$. One single-center study that investigated the incidence of infections and their causative pathogens during the first three months after renal transplantation analyzed 174 renal transplanted patients and detected 51 UTI episodes in 43 patients. The most frequent pathogen, as in the present study, was K. pneumoniae, followed by E. faecalis and E. $\operatorname{coli}^{(22)}$.

The antimicrobial susceptibility profile of strains in the present study showed a very worrying finding: a high percentage of the K. pneumoniae strains were highly resistant to carbapenem antibiotics. 
TABLE 2 - Identified microorganisms in all 320 (17.3\%) positive urine cultures from 1,847 renal transplant patients during the first year after surgery. Ribeirão Preto, Brazil, 2014.

\begin{tabular}{lc}
\hline Microorganisms & $\begin{array}{c}\text { Number of urine culture } \\
\text { samples }\end{array}$ \\
\hline
\end{tabular}

Gram negative $-79.8 \%$

Klebsiella pneumoniae SC

Klebsiella pneumoniae CR

Subtotal

Escherichia coli SC

Subtotal

Pseudomonas aeruginosa SC

Pseudomonas aeruginosa CR

Subtotal

Acinetobacter baumannii SC

$\begin{array}{cc}5 & 1.4 \\ 5 & 1.4 \\ 36 & 10.6 \\ 2 & 0.6 \\ 38 & 11.2\end{array}$

Subtotal

Gram positive - 15.1\%

Enterococcus sp. *

Enterococcus sp. \&

Subtotal

Staphylococcus sp."

44

Staphylococcussp.@

Subtotal

\section{Fungus $-5.1 \%$}

Candida albicans

4

1.1

Candida sp

Subtotal

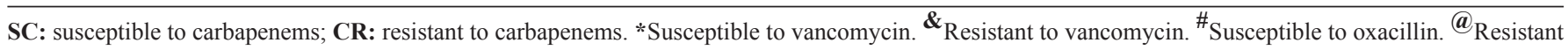
to oxacillin.

The most frequent pathogens associated with UTIs in this facility have changed in recent years. One study performed at this center from 1995-1996 showed that the most frequent pathogen was $S$. marcescens, which was susceptible to the majority of antibiotics tested, instead of K. pneumonia, which is more prevalent currently. Furthermore, none of the strains in previous reports showed resistance to carbapenem antibiotics ${ }^{(23)}$. We speculate that the emergence of these drug-resistant strains is associated with the indiscriminate use of antibiotics as well as the increasing number of more severe cases admitted to the hospital. These drug-resistant strains are widespread globally and represent a challenge to the control and management of infections, especially in patients with solid organ transplantation.

In similar populations in other parts of the world, microorganisms have shown different resistance patterns and even an absence of pathogens resistant to carbapenem antibiotics. Vidal et al. ${ }^{(16)}$ reported $E$. coli extended spectrum beta-lactamase (ESBL) producers in $26.3 \%$ of their samples. In addition, quinolone resistance was identified in $38 \%$ of E. coli isolates, $31 \%$ of Klebsiella spp. isolates, and $21 \%$ of 


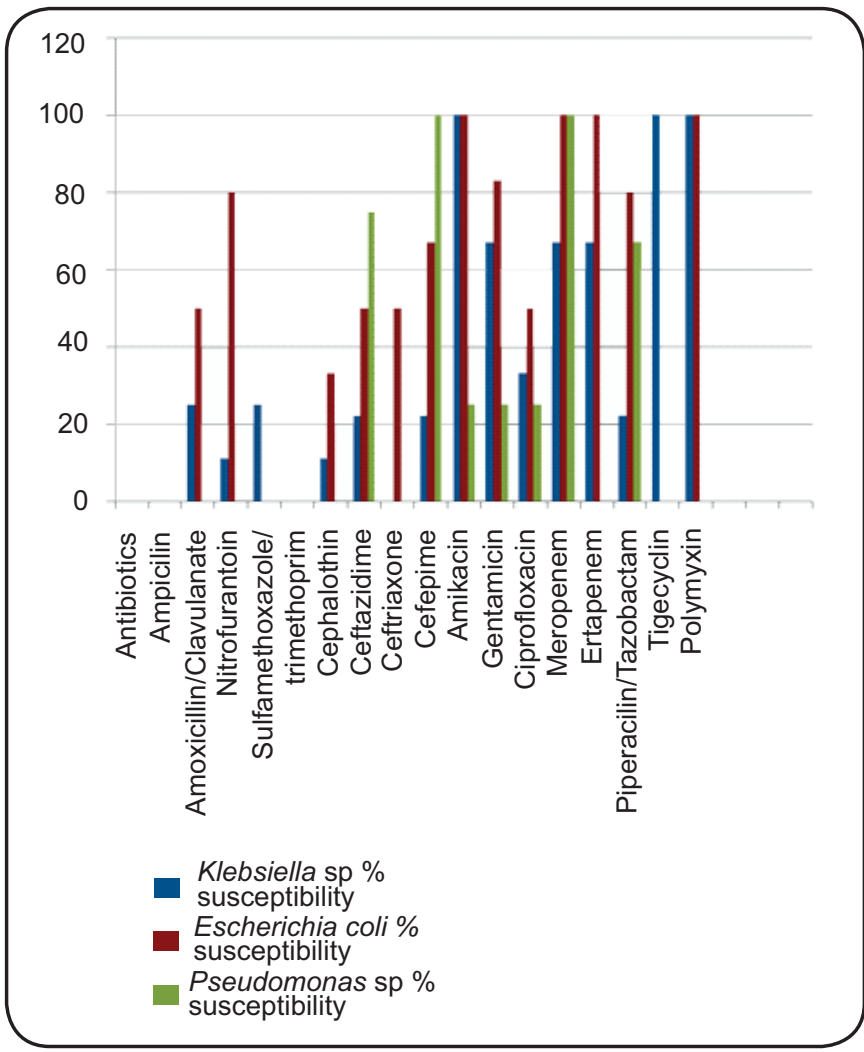

FIGURE 1 - Susceptibility rates of the most frequently isolated microorganisms from renal transplant patients with urinary tract infections. Ribeirão Preto, Brazil, 2014.

Pseudomonas aeruginosa spp. isolates ${ }^{(16)}$. Golebiewska et al. ${ }^{(24)}$ showed that the most frequently isolated microorganisms were E. coli, E. faecium, Klebsiella spp. and Proteus spp; ESBL strains were present in $32 \%$ of these urine cultures ${ }^{(24)}$. In another study, the incidence of ESBL strains was 34\%, with three cases of multi-resistant strains of Klebsiella spp. and two cases of Pseudomonas spp. ${ }^{(25)}$. Other authors have identified $26.3 \%$ of UTIs to be caused by K. pneumoniae carbapenemase-producing strains in renal transplant patients ${ }^{(26)}$. One study performed in an Italian teaching hospital over 7 months demonstrated an increase in multi-resistant bacteria strains; in the samples that showed $K$. pneumoniae, the urinary tract was the most frequent infection site $^{(27)}$. In 2008, the Centers for Disease Control and Prevention (CDC) published that in 2000 , only $1 \%$ of $K$. pneumoniae isolates produced carbapenemases as compared to $8 \%$ in $2007^{(28)}$. In 2007, the Brazilian Society of Infectious Disease (SBI), due to an increasing number of Enterobacteriaceae showing multidrug resistance (especially increasing numbers of $K$. pneumoniae strains showing resistance to carbapenem antibiotics), emphasized that the combination of drugs such as polymyxin (B or E) with carbapenems or aminoglycosides may show better results ${ }^{(29)}$. Another suggested drug combination was polymyxin (B or E) with tigecycline for blood stream infections ${ }^{(29)}$.

At least one UTI episode was observed in $26 \%$ of renal transplant patients during the first year post-transplantation.
Among the UTI risk factors studied, the only one that was associated with a higher incidence of infection was female sex. There were no deaths associated with UTIs in our study. The most frequently isolated microorganism was $K$. pneumoniae, with $33 \%$ of the strains resistant to carbapenem antibiotics.

\section{CONFLICT OF INTEREST}

The authors declare that there is no conflict of interest.

\section{REFERENCES}

1. Kasper DL, Fauci AS, Longo DL, Braunwald E, Hauser SL, Jameson JL. Harrison Medicina Interna. 17 $7^{\text {th }}$ ed. Rio de Janeiro: McGraw Hill; 2008.

2. Kosmadakis G, Daikos GL, Pavlopoulou ID, Gobou A, Kostakis $\mathrm{A}$, Tzanatou-Exarchou $\mathrm{H}$, et al. Infectious complications in the first year post renal transplantation. Transplant Proc 2013; 45:1579-1583.

3. García-Prado ME, Cordero E, Cabello V, Pereira P, Torrubia FJ, Ruíz M, et al. Infectious complications in 159 consecutive kidney transplant recipients. Enferm Infec Microbiol Clin 2009; 27:22-27.

4. Memikoğlu KO, Keven K, Sengüi S, Soypaçaci Z, Ertürk S, Erbay B. Urinary tract infections following renal transplantation: a single-center experience. Transplant Proc 2007; 39:3131-3134.

5. Barbouch S, Cherif M, Ounissi M, Karoui C, Mzoughi S, Hamida $\mathrm{FB}$, et al. Urinary tract infections following renal transplantation: a single-center experience. Saudi J Kidney Dis Transpl 2012; 23:1311-1314.

6. Rubin RH. Infectious disease complications of renal transplantation. Kidney Int 1993; 44:221.

7. Rubin RH, Wolfson JS, Cosimi AB, Tolkoff-Rubin NE. Infection in the renal transplant recipient. Am J Med 1981; 70:405-411.

8. Lyerová L, Lácha J, Skibová J, Teplan V, Vítko S, Schück O. Urinary tract infection in patients with urological complications after renal transplantation with respect to long-term function and allograft survival. Ann Transplant 2001; 6:19-20.

9. Wilson CH, Bhatti AA, Rix DA, Manas DM. Routine intraoperative ureteric stenting for kidney transplant recipients. Cochrane Database Syst Rev 2005; 19:CD004925.

10. Souza RM, Olsburgh J.Urinary tract infection in the renal transplant patient. Nat Clin Pract 2008; 4:252-264.

11. Rao KV, Andersen RC. Long-term results and complications in renal transplant recipients. Observations in the second decade. Transplantation 1988; 45:45.

12. Grekas D, Thanos V, Dioudis C, Alivanis P, Tourkantonis A. Treatment of urinary tract infections with ciprofloxacin after renal transplantation. Int J Clin Pharmacol Ther Toxicol 1993; 31:309-311.

13. Dharnidharka VR, Agodoa LY, Abbott KC. Effects of urinary tract infection on outcomes after renal transplantation in children. Clin J Am Soc Nephrol 2007; 2:100-106.

14. Brooks J. Using the National Healthcare Safety Network for CAUTI Surveillance. Centers for Disease Control and Prevention (CDC). Division of Healthcare Quality Promotion; 2012.

15. Clinical and Laboratory Standards Institute (CLSI). Performance Standards for Antimicrobial Disk Susceptibility Tests; Approved Standard. $11^{\text {th }}$ ed. CLSI document M02-A11. Wayne, Pennsylvania, USA: CLSI; 2012. 
16. Vidal E, Torre-Cisneros J, Blanes M, Montejo M, Cervera C, Aguado JM, et al. Bacterial urinary tract infection after solid organ transplantation in the RESITRA cohort. Transpl Infect Dis 2012; 14: 595-603.

17. Khanna P, Abraham G, Mohamed Ali AA, Miriam PE, Mathew $\mathrm{M}$, Lalitha MK, et al. Urinary tract infections in the era of newer immunosuppressant agents: a tertiary care center study. Saudi J Kidney Dis Transpl 2010; 21:876-880.

18. Chuang P, Parikh CR, Langone A. Urinary tract infections after renal transplantation: a retrospective review at two US transplant centers. Clin Transplant 2005: 19:230-235.

19. Sousa SR, Galante NZ, Barbosa DA, Pestana JAM. Incidence of infectious complications and their risk factors in the first year after renal transplantation. J Bras Nefrol 2010; 32:77-84.

20. Lu PL, Liu YC, Toh HS, Lee YL, Liu YM, Ho CM, et al. Epidemiology and antimicrobial susceptibility profiles of Gram-negative bacteria causing urinary tract infections in the Asia-Pacific region: 20092010 results from the Study for Monitoring Antimicrobial Resistance Trends (SMART). Int J Antimicrob Agents 2012; 40:37-43.

21. Kawecki D, Kwiatkowski A, Sawicka-Grzelak A, Durlik M, Paczek L, Chmura A, et al. Urinary tract infections in the early posttransplant period after kidney transplantation: etiologic agents and their susceptibility. Transplant Proc 2011; 43:2991-2993.

22. Lyerová L, Viklický O, Nemcová D, Teplan V. The incidence of infectious diseases after renal transplantation: a single-centre experience. Int J Antimicrob Agents 2008; 31:S58-S62.

23. Moysés-Neto M, Costa RS, Reis MA, Ferraz AS, Saber LT, Batista ME, et al. Use of ciprofloxacin as a prophylatic agent in urinary infections in renal transplant recipients. Clin Transplant 1997; 11:446-452.

24. Golebiewska J, Debska-Slizien A, Komarnicka J, Samet A, Rutkowski B. Urinary tract infections in renal transplant recipients. Transplant Proc 2011; 43:2985-2990.

25. Parapiboon W, Ingsathit A, Jirasiritham S, Sumethkul V. High incidence of bacteriuria in early post-kidney transplantation; results from a randomized controlled study. Transplant Proc 2012; 44:734-736.

26. Bergamasco MD, Barbosa MB, Garcia DO, Cipullo R, Moreira JC, Baia C, et al. Infection with Klebsiella pneumoniae carbapenemase (KPC)-producing K. pneumoniae in solid organ transplantation. Transpl Infect Dis 2012; 14:198-205.

27. Gaibani P, Ambretti S, Berlingeri A, Cordovana M, Farruggia $\mathrm{P}$, Panico M, et al. Rapid increase of carbapenemase-producing Klebsiella pneumoniae strains in a large Italian hospital: surveillance period. Eurosurveillance 2011; 16(8):pii:19800.

28. Center for Disease Control and Prevention (CDC). Laboratory Protocol for Detection of Carbapenen-resistant or carbapenemaseproducing, Klebsiella sp and E. coli from Retal Swabs. Atlanta, USA: CDC; 2009.

29. Sociedade Brasileira de Infectologia (SBI) (Internet). Comitê de Bacteriologia Clínica da SBI. Consulta pública. Sugestões para o Tratamento das Infecções Causadas por Enterobactérias Resistentes aos Carbapenêmicos. 2011. (Cited 2013 March). Available at: http://www.infectologia.org.br/anexos/Consulta $\% 20$ publica_comite $\% 20$ bacteriologia $\% 20$ clinica_KPC_marco $\% 20$ 2011.pdf 